\title{
The Crucial Role of Error Correlation for Uncertainty Modeling of CFD-Based Aerodynamics Increments
}

\author{
Michael J. Hemsch ${ }^{*}$ and Eric L. Walker ${ }^{\dagger}$ \\ NASA Langley Research Center, Hampton, VA, 23681
}

\begin{abstract}
The Ares I ascent aerodynamics database for Design Cycle 3 (DAC-3) was built from wind-tunnel test results and CFD solutions. The wind tunnel results were used to build the baseline response surfaces for wind-tunnel Reynolds numbers at power-off conditions. The CFD solutions were used to build increments to account for Reynolds number effects. We calculate the validation errors for the primary CFD code results at wind tunnel Reynolds number power-off conditions and would like to be able to use those errors to predict the validation errors for the CFD increments. However, the validation errors are large compared to the increments. We suggest a way forward that is consistent with common practice in wind tunnel testing which is to assume that systematic errors in the measurement process and/or the environment will subtract out when increments are calculated, thus making increments more reliable with smaller uncertainty than absolute values of the aerodynamic coefficients. A similar practice has arisen for the use of CFD to generate aerodynamic database increments. The basis of this practice is the assumption of strong correlation of the systematic errors inherent in each of the results used to generate an increment. The assumption of strong correlation is the inferential link between the observed validation uncertainties at wind-tunnel Reynolds numbers and the uncertainties to be predicted for flight. In this paper, we suggest a way to estimate the correlation coefficient and demonstrate the approach using code-to-code differences that were obtained for quality control purposes during the Ares I CFD campaign. Finally, since we can expect the increments to be relatively small compared to the baseline response surface and to be typically of the order of the baseline uncertainty, we find that it is necessary to be able to show that the correlation coefficients are close to unity to avoid overinflating the overall database uncertainty with the addition of the increments.
\end{abstract}

\section{Nomenclature \\ $\mathrm{CA}$ \\ CFD \\ CM \\ CN \\ CRM \\ DAC-2A \\ DAC-3 \\ $x$ \\ $y$ \\ $y_{C F D}(x)$ \\ $y_{\text {EXP }}(x)$ \\ forebody axial force coefficient normalized by the nominal cross-sectional area of the first stage computational fluid dynamics pitching moment coefficient in missile axes normalized by the nominal diameter and cross- sectional area of the first stage \\ normal-force coefficient in missile axes normalized by the nominal cross-sectional area of the first stage \\ rolling-moment coefficient in missile axes normalized by the nominal diameter and cross-sectional area of the first stage \\ Design Cycle 2-A for the Ares I ascent configuration (full stack) \\ Design Cycle 3 for the Ares I ascent configuration (full stack) \\ a vector of independent variables in the inference space of interest \\ a vector of dependent variables \\ the CFD result at $x$ where $y$ is a vector composed of the six force and moment components in missile axes. \\ the experimental result at $x$ where $y$ is a vector composed of the six force and moment components in missile axes.}

\footnotetext{
* Aerospace Engineer, Configuration Aerodynamics Branch, Mail Stop 499, Associate Fellow.

${ }^{\dagger}$ Aerospace Engineer, Configuration Aerodynamics Branch, Mail Stop 267, Senior Member.
} 


$\begin{array}{ll}\alpha_{T} & \text { total angle of attack in missile coordinates } \\ \varepsilon_{y}(x) & \text { a random variable equal to the difference } y_{C F D}(x)-y_{E X P}(x), \text { see eq.(1) } \\ \varphi & \text { aerodynamic roll angle in missile coordinates }\end{array}$

\section{Introduction}

For various reasons, including cost and time constraints as well as suitable wind tunnel availability, the Ares I DAC3 aero databases for the ascent configuration were built using both wind tunnel and computational fluid dynamics (CFD) data. ${ }^{1}$ Tests were conducted in the Boeing Polysonic Wind Tunnel for the Mach range of 0.5 to 1.6 and the NASA Langley Unitary Plan Wind Tunnel for the Mach range of 1.6 to 4.5. The wind tunnel results were used to build the baseline response surfaces for wind-tunnel Reynolds numbers and power-off conditions for the roll-control motors. The wind tunnel test efforts are discussed in references 2 and 3 . The uncertainty modeling process for the DAC-3 baseline response surfaces used the same approach described for an earlier design configuration (DAC-2B). ${ }^{4}$ An artist's sketch of the Ares I vehicle is shown in figure 1. Note that the vehicle is covered with numerous protuberances. Currently, there are over 40 protuberances, each one of which acts as a blunt fin. There are also numerous axisymmetric steps and rings. The complex geometry of the vehicle requires tens of millions of grid points and tens of thousands of iterations to resolve the local flow physics sufficiently for CFD analysis. The baseline code used for the calculations is the unstructured-grid solver USM3D. ${ }^{5-7}$ The alternate code used for codeto-code comparisons is the overset-structured-grid code Overflow. ${ }^{8,9}$

At the beginning of the effort, it was not clear just how large the two types of increments (Reynolds number and power-on/off) would be relative to the baseline. For the Ares project, the flight Reynolds numbers are typically one to two orders-of-magnitude larger in the high dynamic pressure range of a typical trajectory than those of the wind tunnel tests. The Reynolds number power-off increments turned out to be roughly the size of the baseline uncertainties. One would hope that uncertainty modeling for the CFD solutions would give values smaller than the increments which would make their uncertainties relatively insignificant. However, for the Ares I DAC-3 aero database, the validation errors obtained from comparing the USM3D results to the experimental data are much larger than the CFD-derived Reynolds number increments, as shown in the following sections.

An approach different from having to report the relatively large validation errors for the increments is suggested from typical aerodynamic testing and analysis. For a given wind-tunnel test, it is common practice to assume that systematic errors in the measurement process or the environment will essentially subtract out when increments are used, thus making increments more accurate than absolute values of the aero coefficients. The basis of this practice is, of course, the assumption of strong correlation of the systematic errors inherent in each of the results used to generate an increment. However, to the authors' knowledge, no quantitative evidence of the validity of the strong correlation assumption for wind-tunnel testing has been made. At least one metrology author ${ }^{10}$ has made a plea for obtaining the data necessary to evaluate such correlations. But the same author points out that it is expensive to do so due to the need to obtain multiple sets of data at identical conditions. In fact, for each data point in an error correlation plot, four data points are needed. Nevertheless, it is tempting to adopt a similar practice for CFD-derived aero increments. But such a practice has even less underpinning currently than the wind tunnel practice.

The plan of the rest of the paper is as follows:

(1) We display the Reynolds number power-off increments for the Ares I DAC-3 aero database.

(2) We show the validation errors obtained by comparing the CFD solutions at wind tunnel Reynolds numbers to wind tunnel data that have been adjusted to remove systematic errors.

(3) We discuss a CFD error prediction model developed by Easterling ${ }^{11}$ and extend it to the estimation of increment uncertainty.

(4) We provide code-to-code comparisons (USM3D - Overflow) obtained during the CFD campaign for quality control purposes.

(5) We use the code-to-code comparisons to estimate correlation coefficients and show that the correlation coefficients are not necessarily close to unity.

(6) We suggest an approach for further wind-tunnel testing and CFD campaigns to evaluate and predict the necessary correlation coefficients. 


\section{Ares I Ascent Reynolds Number Increments}

The actual vehicle flight trajectories encounter Reynolds numbers that are one to two orders of magnitude larger than those encountered in the wind tunnel tests. Since it is currently not possible to do ground tests at the actual flight Reynolds numbers, the decision was made to use CFD to predict the aero increments due to the Reynolds number differences. The computed Reynolds number increments for the DAC-3 ascent configuration are shown in figures 2(a), 3(a), 4(a), and 5(a) for the normal-force coefficient, pitching-moment coefficient, axial-force coefficient, and rolling-moment coefficient, respectively, in missile-axis coordinates. The increments are shown as functions of Mach number with the total angle of attack $\left(\alpha_{T}\right)$ and the aerodynamic roll angle $(\varphi)$ as parameters. In other words, each point in the figures corresponds to a particular Mach number, $\alpha_{T}$ and aerodynamic roll angle. Thus, the variation of the increments due to $\alpha_{T}$ and $\varphi$ is pooled at each Mach number. The Mach number range is 0.5 to 4.5 ; the total angle of attack range is 0 to 8 degrees; the roll angle range is 0 to 360 degrees. Roughly, the wind-tunnel-to-flight effect is predicted to decrease $\mathrm{CN}, \mathrm{CM}$ and $\mathrm{CA}$, depending on the specific total angle of attack and roll angle values. The aero-induced rolling moment is predicted to increase somewhat. These trends are expected because of the decreased thickness of the vehicle boundary layer at the flight conditions.

We point out that the wind-tunnel baseline database has roughly 3500 cardinal points. It would be prohibitive to make that many CFD runs for both Reynolds numbers, so a much small subset is always used together with a model of the effect to reasonably cover all of the conditions.

\section{Validation Errors}

A subset of the acquired wind tunnel data was used for comparison with the USM3D results. Following the procedures described in reference 4, the full-protuberance data were corrected for systematic errors using the data from an axisymmetric version of the full-protuberance model (protuberances removed). The corrected results were compared with the available USM3D calculations to obtain validation errors (USM3D - experiment). The validation errors are shown in figures 2(b), 3(b), 4(b) and 5(b). The plot scales are the same as for the Reynolds number increment plots but with shifted origins. It can be readily seen that the ranges of the validation errors for individual runs are substantially larger than the ranges of the computed Reynolds number increments. Unless the validation errors are at least somewhat positively correlated, the validation component of the uncertainty associated with the Reynolds number increment would be a large or larger that the wind-tunnel baseline database uncertainty.

\section{Extension of Easterling's Error Prediction Model to Increments}

Easterling ${ }^{11}$ has discussed the need to measure the predictive capability of a computational model. To quote Easterling directly:

\section{A critical inferential link is required to connect observed prediction errors in experimental contexts to bounds on prediction errors in untested applications... Model validation experiments should be designed and conducted in ways that permit a realistic estimate of prediction errors...in application environments.}

Easterling wrote the following equation

$$
y_{C F D}(x)=y_{E X P}(x)+\varepsilon_{y}(x)
$$

where $x$ is a vector of independent variables in the inference space of interest. The vector $y_{C F D}(x)$ is the CFD result at $x$ where, in our case, $y$ is a vector composed of the six force and moment components in missile axes. The vector $y_{E X P}(x)$ is the experimental result at $x$. For the purposes of the discussion in this paper, we will assume that the experimental error associated with $y_{E X P}(x)$ is much smaller than $\varepsilon_{y}(x)$. The vector $\varepsilon_{y}(x)$ is a random variable with an unknown distribution. Easterling pointed out that, although $\varepsilon_{y}(x)$ could be estimated for available test conditions, the real need is for $\varepsilon_{y}$ at the prediction conditions. In other words, it is necessary to find a way to predict $\varepsilon_{y}$ at the conditions of actual interest. And the key is to find a suitable inferential link.

The actual equation of interest for this paper is, however, 


$$
\Delta y_{C F D}(x)=\Delta y_{E X P}(x)+\varepsilon_{\Delta y}(x)
$$

since we are discussing increments. Thus, we will have to somehow model $\varepsilon_{\Delta y}(x)$.

We rewrite eq.(2) as

$$
\begin{aligned}
\varepsilon_{\Delta y} & =\Delta y_{C F D}-\Delta y_{E X P} \\
& =\left(y_{C F D, 2}-y_{C F D, 1}\right)-\left(y_{E X P, 2}-y_{E X P, 1}\right)
\end{aligned}
$$

where the dependence on $x$ has been suppressed for convenience. The subscript "1" refers to the baseline condition while subscript " 2 " refers to the prediction condition of interest. For example, condition 1 could refer to the Reynolds number at which the wind tunnel tests were run while condition 2 could be the flight Reynolds number. Rearranging eq.(3) and substituting eq.(1), written for both conditions, into the result gives

$$
\begin{aligned}
\varepsilon_{\Delta y} & =\left(y_{C F D, 2}-y_{E X P, 2}\right)-\left(y_{C F D, 1}-y_{E X P, 1}\right) \\
& =\varepsilon_{y 2}-\varepsilon_{y 1}
\end{aligned}
$$

It is clear now that the error distribution of interest, $\varepsilon_{\Delta y}(x)$, is given by the difference between the error distributions for conditions 1 and 2. Hence, the magnitude of $\varepsilon_{\Delta y}(x)$ will depend on the correlation of $\varepsilon_{y 2}$ with $\varepsilon_{y 1}$. And we want to be able to predict that correlation with some confidence.

Eq.(4) suggests the inferential link needed for CFD increment prediction given validation information for individual coefficients; that is, we can see that the means must be nearly equal and the scatter terms must be positively correlated for the increment errors to be significantly smaller than the validation errors that we have seen for the current example.

\section{Code-to-Code Comparisons}

Results from the baseline code were compared for quality control purposes during the CFD campaign with the alternate code $\left(\right.$ Overflow $\left.^{8,9}\right)$. The two codes used the same turbulence model but have different discretization approaches (unstructured vs overset-structured) and different solution strategies. The code-to-code comparisons that are available for the DAC-3 campaign and a previous campaign (DAC-2A) are shown in figures 2(c), 3(c), 4(c) and 5(c). The comparisons are shown as code-to-code differences (Overflow - USM3D) as a function of Mach number with $\alpha_{T}$ and $\varphi$ as parameters. The scales are the same as for figures 2(a), 3(a), 4(a) and 5(a) but with shifted vertical origins. It can be seen that the ranges of the code-to-code differences are considerably larger than the ranges of the Reynolds number increments for CN, CA and CRM and are roughly the same size for CM. Below we will use the code-to-code comparisons in correlation plots to estimate correlation coefficients.

\section{Estimation of Correlation Coefficients for the Code-to-Code Differences}

Now we will derive a useful relationship between the distributions associated with the three terms of eq.(4). As long as we can reasonably assume that the terms in eq.(4) are random variables, we can write the following expression relating the variances of the terms ${ }^{12}$

$$
\sigma_{\varepsilon_{\Delta y}}^{2}=\sigma_{\varepsilon_{y 2}}^{2}-2 \rho \sigma_{\varepsilon_{y 1}} \sigma_{\varepsilon_{y 2}}+\sigma_{\varepsilon_{y 1}}^{2}
$$

where $\rho$ is the correlation coefficient. The Ares I project does not currently have the necessary information to evaluate $\rho$ for the validation errors, so we will continue with the example using the code-to-code differences. Further, referring to figures $2(\mathrm{c})-5(\mathrm{c})$, we see that it is reasonable for our discussion to assume that

$$
\sigma_{\varepsilon_{y 2}}=\sigma_{\varepsilon_{y 1}}
$$

This assumption is additionally somewhat justified since similar best practices will have been used for the two conditions. Substituting eq.(6) into eq.(5) gives 


$$
\begin{aligned}
\sigma_{\varepsilon_{\Delta y}}^{2} & =\sigma_{\varepsilon_{y 1}}^{2}-2 \rho \sigma_{\varepsilon_{y 1}} \sigma_{\varepsilon_{y 1}}+\sigma_{\varepsilon_{y 1}}^{2} \\
& =2 \sigma_{\varepsilon_{y 1}}^{2}(1-\rho)
\end{aligned}
$$

or

$$
\sigma_{\varepsilon_{\Delta y}}=[2(1-\rho)]^{1 / 2} \sigma_{\varepsilon_{y 1}}
$$

Values for the coefficient $[2(1-\rho)]^{1 / 2}$ are given in table 1 . For $\rho<0.5$, data are usually considered not to be significantly correlated. ${ }^{13}$ But it can be seen from the table that $\rho$ must be approximately 0.8 or greater to get a substantial error reduction for the increments based on either the code-to-code differences or the validation errors.

The corresponding pairs of code-to-code differences for flight and wind-tunnel Reynolds numbers are plotted in figure 6 for $C N, C M, C A$ and $C R M$. For each coefficient a trend line is calculated and the corresponding correlation coefficient computed. The values for $\rho$ are $0.85,0.70,0.63,0.82$, respectively. The lines of perfect correlation are also shown. Note that the plots of figure 6 do not require the assumption that $\varepsilon_{y}$ be a random variable with a distribution, albeit unknown. However, it seems likely that some other assumption would be required to provide a correlation model in order for the data subset to be reasonably applied to the entire database inference space.

\section{Planning for Error Prediction Estimation}

The purpose of this paper is to suggest an inference process for estimating the uncertainty associated with CFDderived aerodynamic increments. We demonstrated the approach using code-to-code comparisons, although the process of interest would be for validation as well. As with all model predictions, we want to know if the model can predict trends. The same is true for uncertainty. And we have shown that what is crucial is the correlation between the errors at the baseline condition and the errors at the prediction condition. The code-to-code correlations suggest that what is needed is a prediction of the correlation coefficient. Hence, any validation campaign should include the ability to measure the correlation coefficient for conditions that can be obtained. For example, testing in the National Transonic Facility ${ }^{14}$ would allow for obtaining validation correlation coefficients for a portion of the Reynolds number range needed. Then one would feel more confident using those correlation coefficients, together with the validation data obtained, to predict the CFD increment uncertainty.

\section{Final Remarks}

In this paper, we suggest a way to confidently develop uncertainties for CFD-derived increments. We use the Ares I DAC-3 aerodynamic increments for Reynolds number effects (wind-tunnel to flight) to demonstrate the process. We show that the validation errors at wind tunnel Reynolds numbers are large relative to the increments. We also show that the differences between the baseline code (USM3D) and the alternate code (Overflow) are also large relative to the increments. We then used the code-to-code differences to find an inferential link to connect the flight uncertainties to the baseline values. Finally, we note that this inferential process for the uncertainties is essentially the same one that would be used to infer reasonable extrapolation of the nominal increments.

\section{Acknowledgements}

The authors gratefully thank the NASA Langley Research Center's Ares I Test and Analysis Team for supplying the wind-tunnel and CFD results used in the present analysis. In addition, we thank Ms. Heather P. Houlden for supplying the pitch-run-based wind-tunnel results used for the validation analysis. Finally, we thank Dr. Jeremy T. Pinier and Mr. Jeremy L. Hanke for several helpful conversations on error correlation for the Ares aero databases.

\section{References}

1. Hall, Robert M., “Aerodynamic Characterization Overview of a Modern Launch Vehicle”, To be presented at the 49th AIAA Aerospace Sciences Meeting, Orlando 2011.

2. Tomek, William B., "Overview of Experimental Investigations for Ares Launch Vehicle Development", To be presented at the 49th AIAA Aerospace Sciences Meeting, Orlando 2011. 
3. Pinier, Jeremy T., Niskey, C., "Ares I Aerodynamic Testing at the Boeing Polysonic Wind Tunnel”, To be presented at the 49th AIAA Aerospace Sciences Meeting, Orlando 2011.

4. Hemsch, Michael J., Hanke, Jeremy L., Walker, Eric L., Houlden, Heather P., "Detailed Uncertainty Analysis for Ares I Ascent Aerodynamics Wind Tunnel Database", AIAA-2008-4259, June 2008.

5. Abdol-Hamid, Khalid S., Ghaffari, Farhad, and Parlette, Edward B., "An Overview of Ares-I CFD Ascent Aerodynamic Data Development and Analysis Based on USM3D", To be presented at the 49th AIAA Aerospace Sciences Meeting, Orlando 2011.

6. Pao, S. Paul, "Establishing Approaches to Modeling the Ares I-X and Ares I Roll Control System with Free-Stream Interaction", To be presented at the 49th AIAA Aerospace Sciences Meeting, Orlando 2011.

7. Pandya, Mohagna J., Frink, Neal T., Parlette, Edward B., Samareh, Jamshid A. and Abdol-Hamid, Khaled S., "Enhancements to NASA TetrUSS for Constellation Program", To be presented at the 49th AIAA Aerospace Sciences Meeting, Orlando 2011.

8. Klopfer, Goetz H., Onufer, Jeffrey T., Chan, William N., Pandya, Shisher A., Kless, James, Lee, Henry C., "Analysis of the Ares I Launch Vehicle Stage Separation: Static, Time Accurate, Prescribed Motion, and Coupled 6-DOF Simulations", To be published at the $49^{\text {th }}$ AIAA Aerospace Sciences Meeting, Orlando 2011.

9. Buning, Pieter G., Overflow Home Page, http://aaac.larc.nasa.gov/ buning/, last access April 29, 2010.

10. Grabe, Michael, Measurement Uncertainties in Science and Technology, Springer 2005.

11. Easterling, Robert G., "Measuring the Predictive Capability of Computational Models: Principles and Methods, Issues and Illustrations," Sandia National Laboratories, SAND2001-0243, February 2001.

12. Mandel, John, The Statistical Analysis of Experimental data, Dover, 1964.

13. Taylor, John, An Introduction to Error Analysis: The Study of Uncertainties in Physical Measurement, University Science Books, 1982.

14. Hemsch, Michael J., "Analysis of flow Angularity Repeatability Tests in the NTF", AIAA-2006-0518, January 2006.

Table 1. Values for $\sqrt{2(1-\rho)}$.

\begin{tabular}{|c|c|}
\hline$\rho$ & $\sqrt{2(1-\rho)}$ \\
\hline 0 & 1.41 \\
\hline 0.1 & 1.34 \\
\hline 0.2 & 1.26 \\
\hline 0.3 & 1.18 \\
\hline 0.4 & 1.10 \\
\hline 0.5 & 1.00 \\
\hline 0.6 & 0.89 \\
\hline 0.7 & 0.77 \\
\hline 0.8 & 0.63 \\
\hline 0.9 & 0.45 \\
\hline 0.95 & 0.32 \\
\hline 0.98 & 0.20 \\
\hline 1 & 0 \\
\hline
\end{tabular}




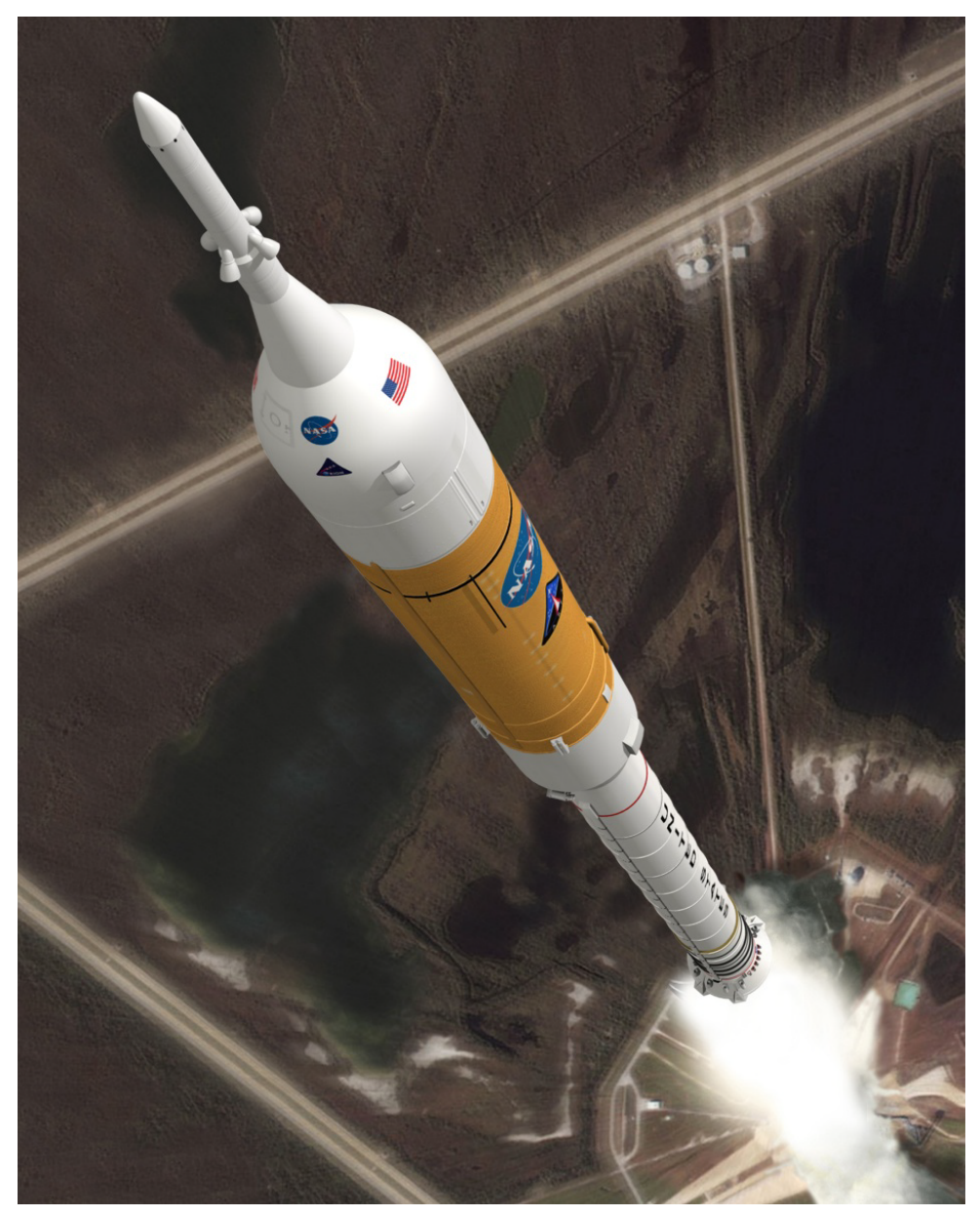

Figure 1. Artist's sketch of an Ares I configuration just after launch. 


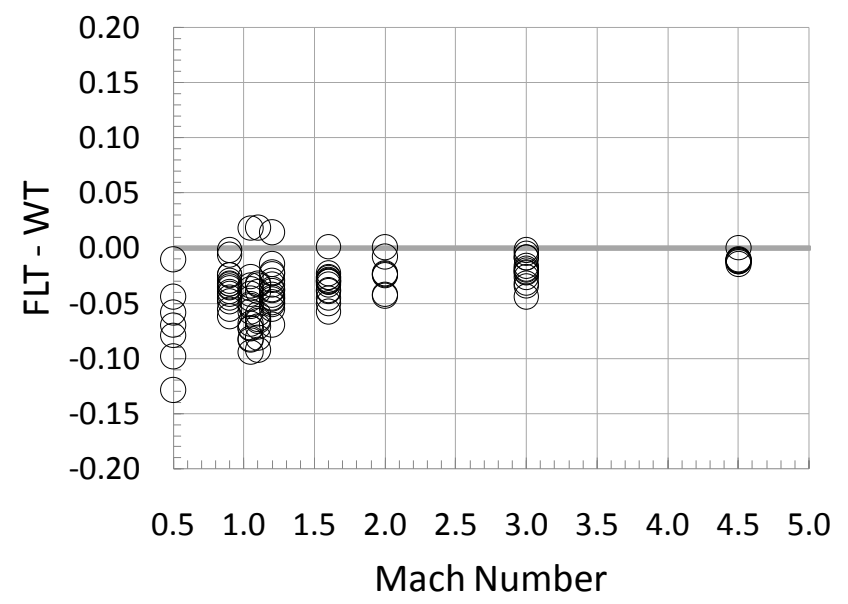

(a) DAC-3 Reynolds Number increments (FLT - WT)

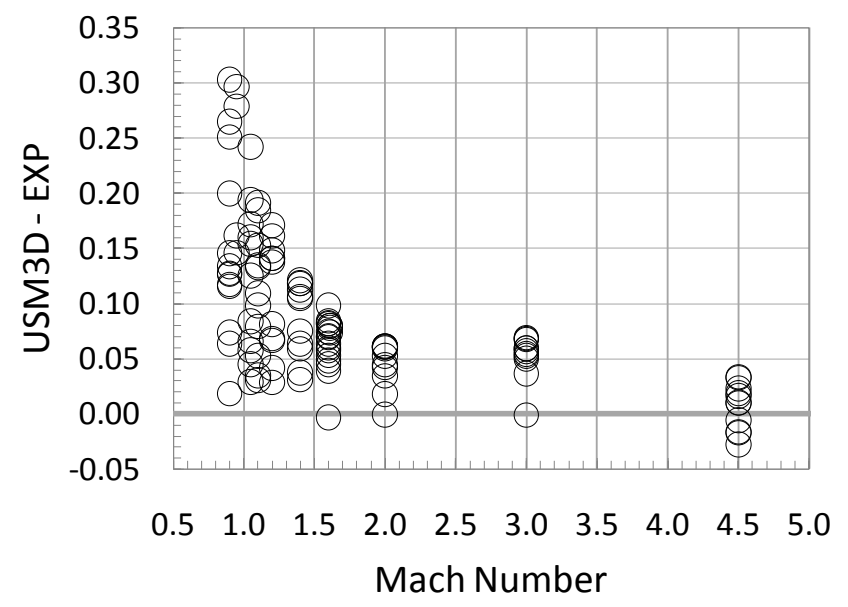

(b) Validation errors (USM3D - EXP)

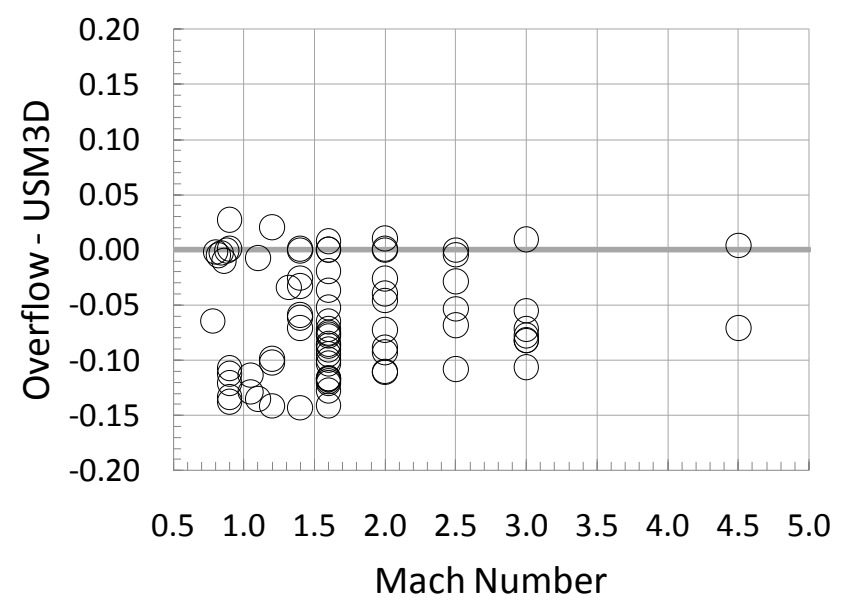

(c) Code-to-code differences (Overflow - USM3D)

Figure 2. CFD Reynolds number increments, validation errors, and code-to-code differences for the normalforce coefficient for various values of the parameters $\alpha_{T}(0$ to $8 \mathrm{deg})$ and $\varphi(0$ to $360 \mathrm{deg})$. 


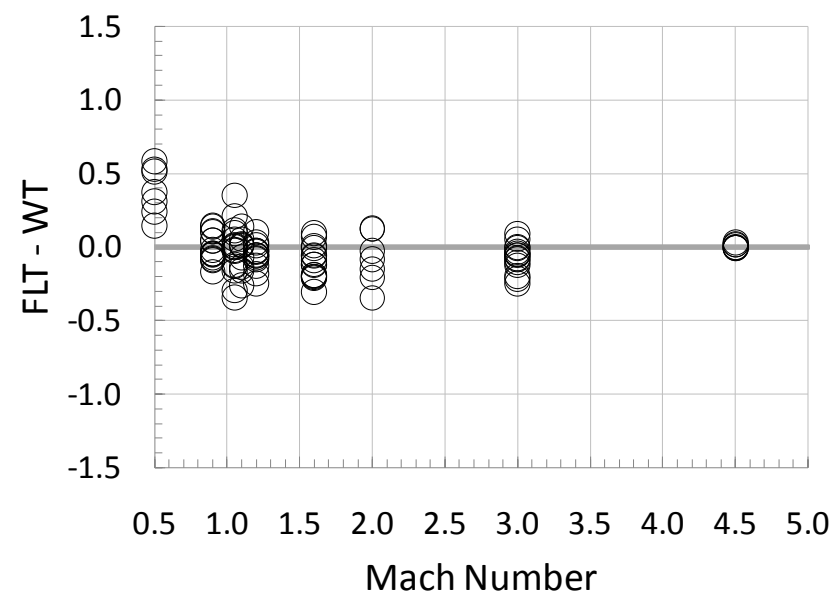

(a) DAC-3 Reynolds Number increments (FLT - WT)

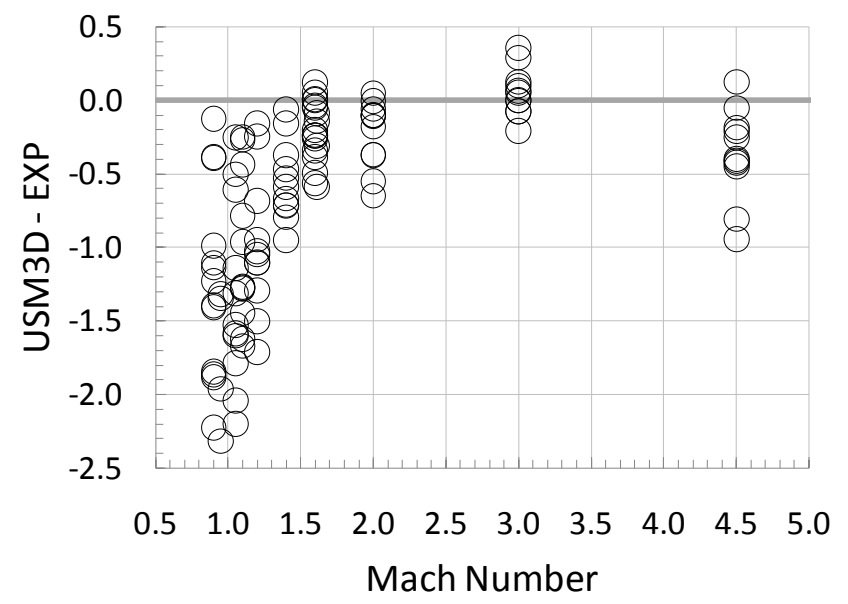

(b) Validation errors (USM3D - EXP)

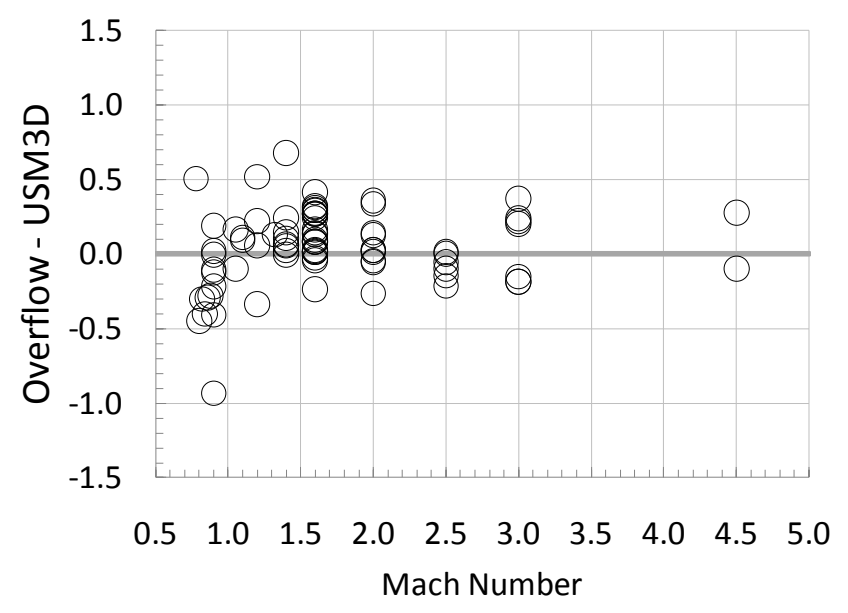

(c) Code-to-code differences (Overflow - USM3D)

Figure 3. CFD Reynolds number increments, validation errors, and code-to-code differences for the pitchingmoment coefficient for various values of the parameters $\alpha_{T}(0$ to $8 \mathrm{deg})$ and $\varphi(0$ to $360 \mathrm{deg})$. 


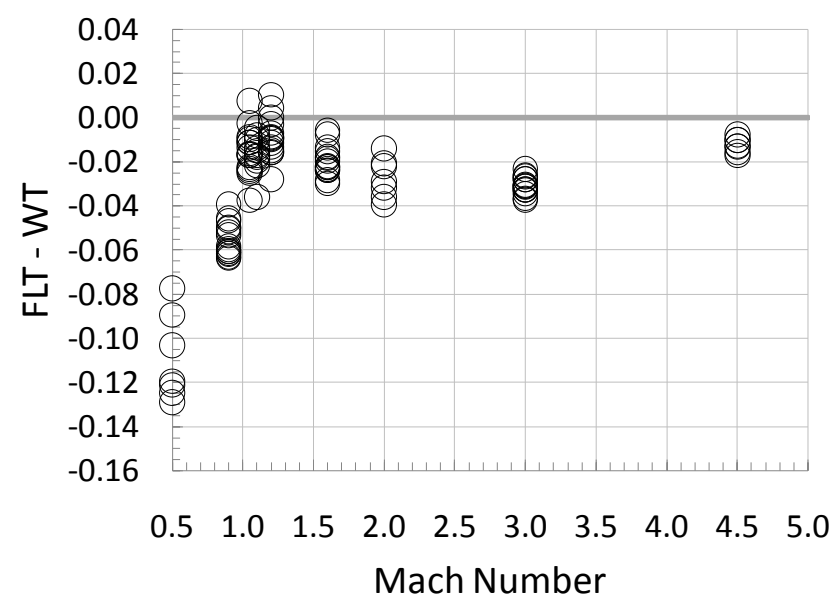

(a) DAC-3 Reynolds Number increments (FLT - WT)

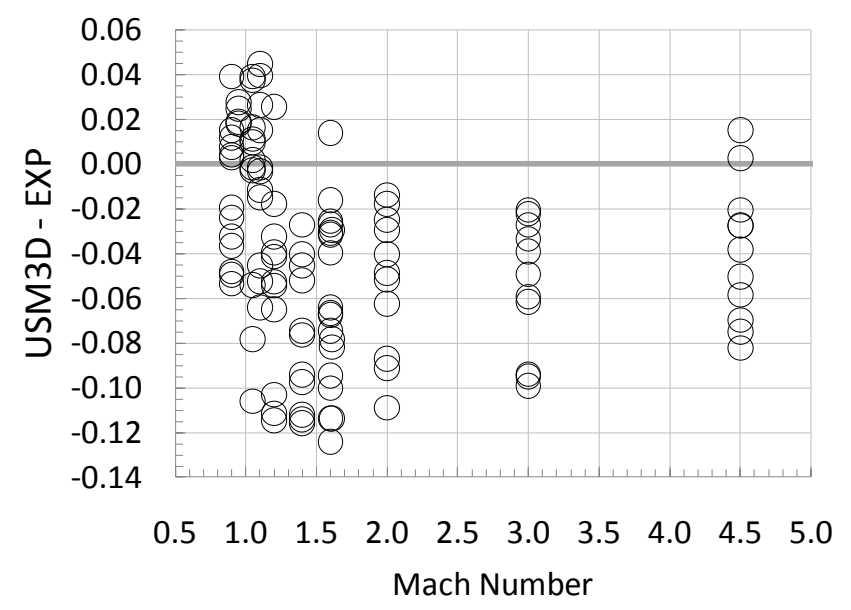

(b) Validation errors (USM3D - EXP)

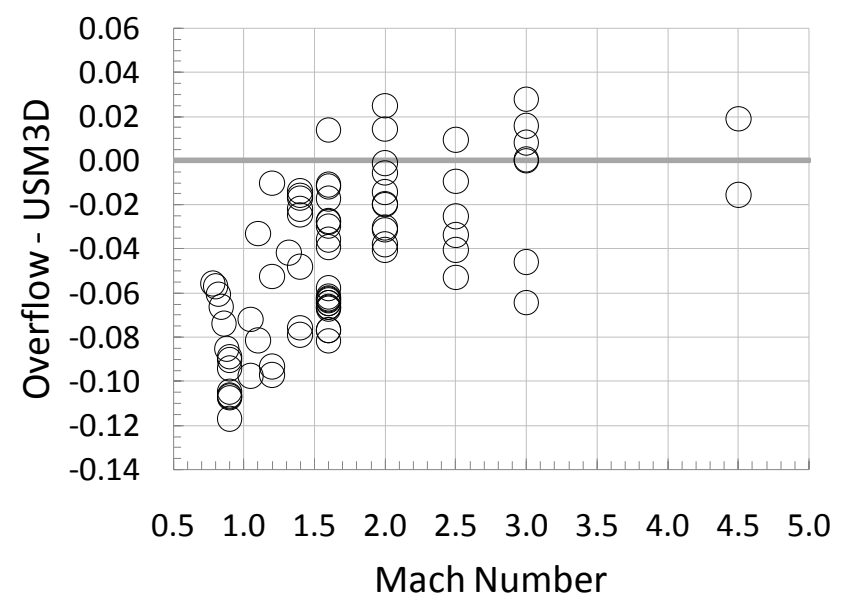

(c) Code-to-code differences (Overflow - USM3D)

Figure 4. CFD Reynolds number increments, validation errors, and code-to-code differences for the axialforce coefficient for various values of the parameters $\alpha_{T}(0$ to $8 \mathrm{deg})$ and $\varphi(0$ to $360 \mathrm{deg})$. 


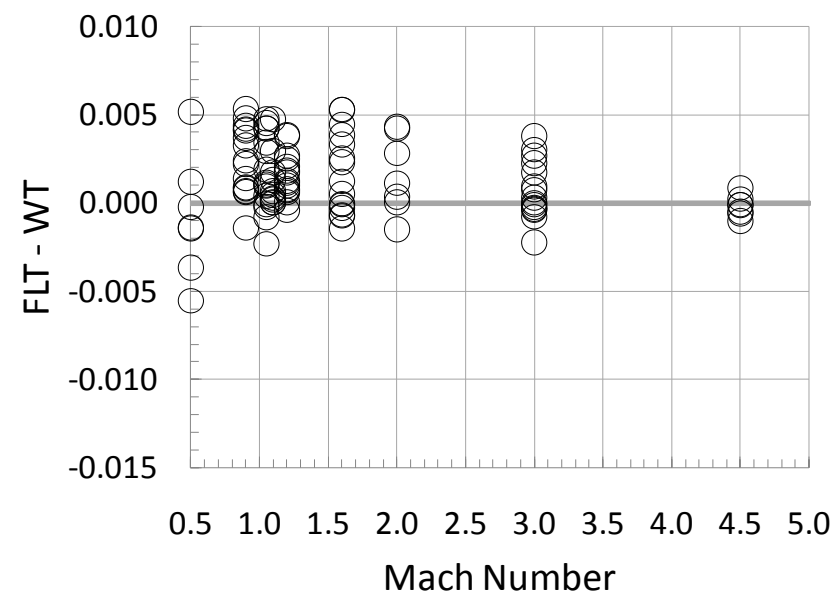

(a) DAC-3 Reynolds Number increments (FLT - WT)

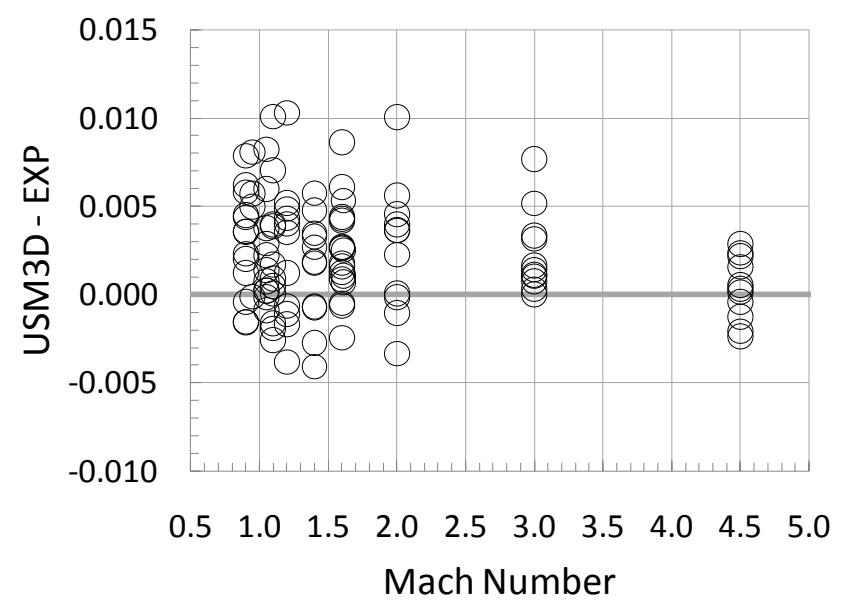

(b) Validation errors (USM3D - EXP)

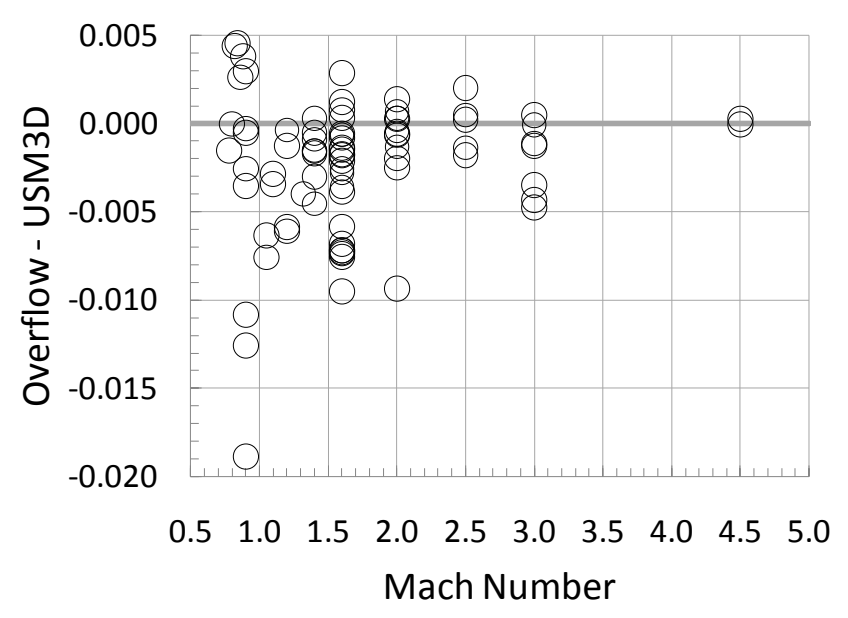

(c) Code-to-code differences (Overflow - USM3D)

Figure 5. CFD Reynolds number increments, validation errors, and code-to-code differences for the rollingmoment coefficient for various values of the parameters $\alpha_{T}(0$ to $8 \mathrm{deg})$ and $\varphi$ ( 0 to $\left.360 \mathrm{deg}\right)$. 


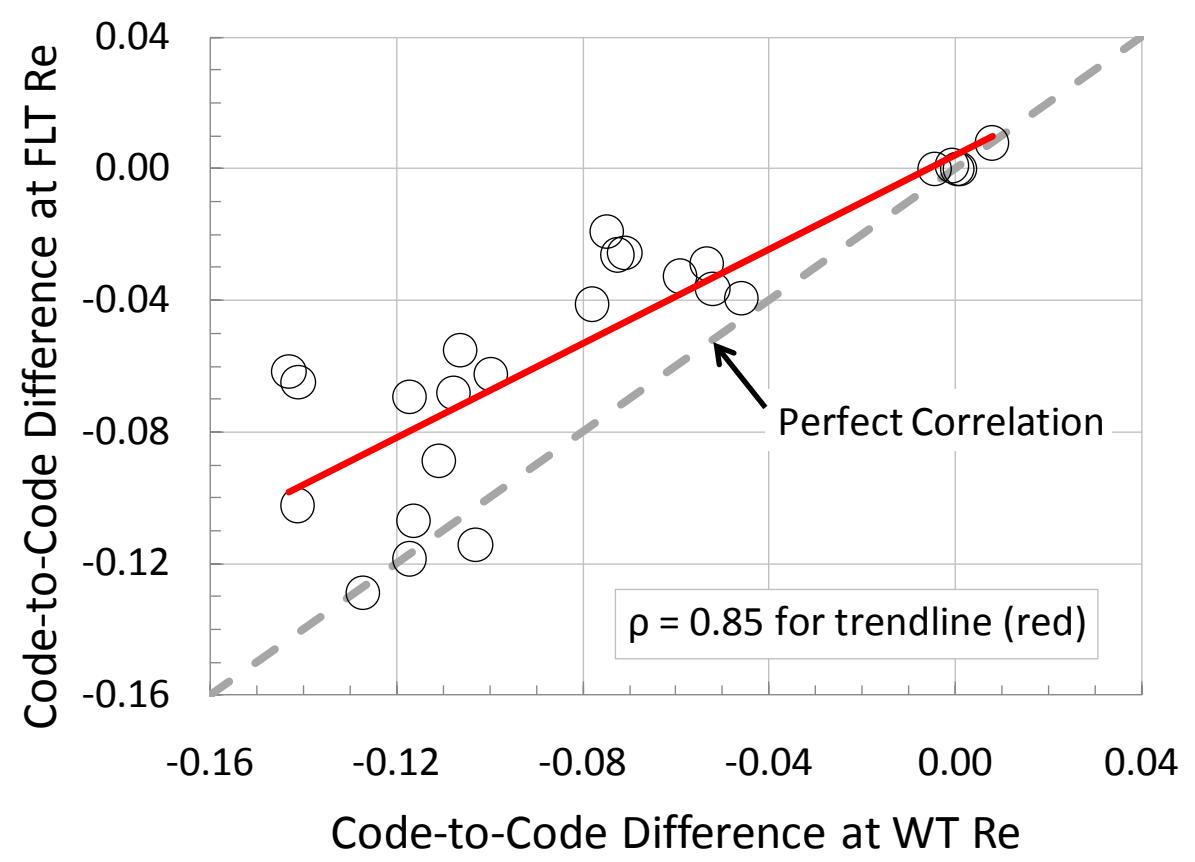

(a) Normal-force coefficient

Figure 6. Correlation of code-to-code errors for $\mathrm{M}=1.6$ for various values of $\alpha_{T}$ and $\varphi$.

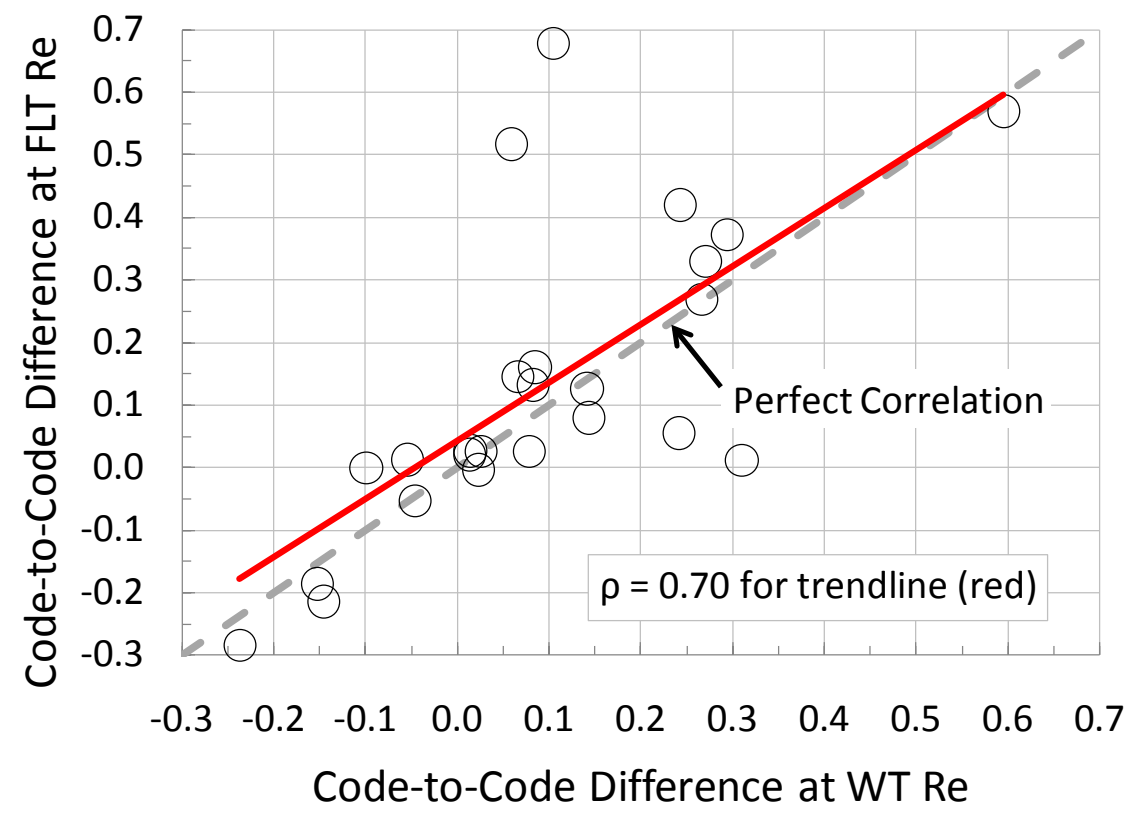

(b) Pitching-moment coefficient

Figure 6. Continued. 


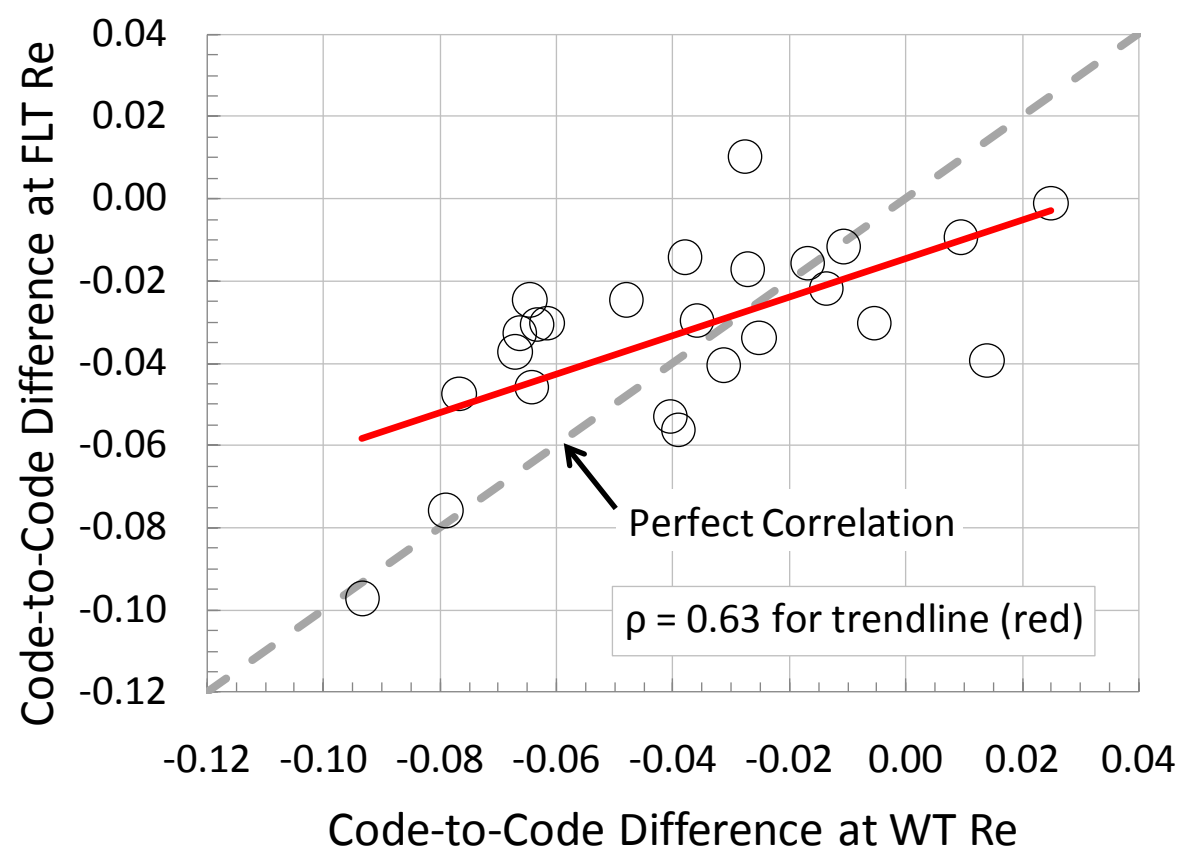

(c) Axial-force coefficient

Figure 6. Continued.

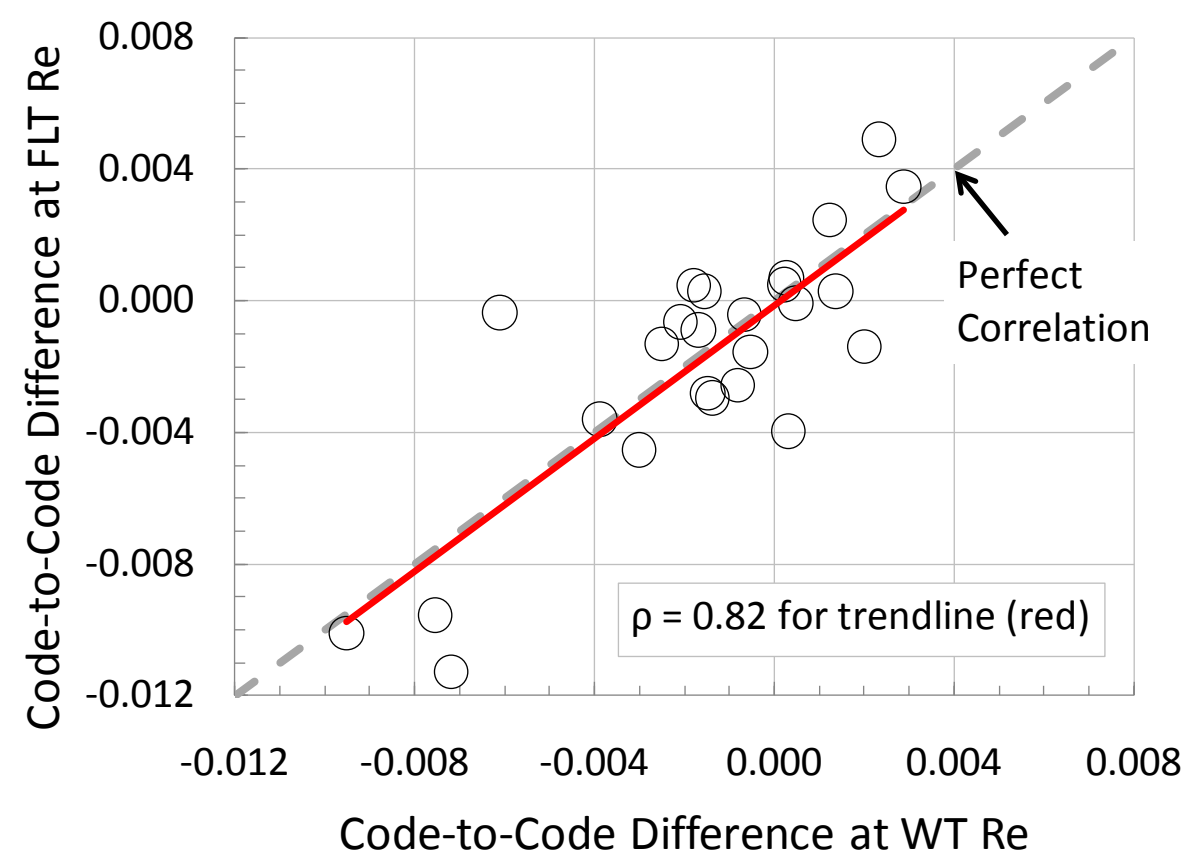

(d) Rolling-moment coefficient

Figure 6. Concluded. 